\title{
Avoiding a chimaera quagmire
}

\author{
Researchers need to take the initiative in addressing a controversial and urgent ethical issue: under what \\ circumstances should the fusion of cells of animals and humans be permitted?
}

ast month, legislative revisions to the UK Human Fertilisation and Embryology Act were proposed in which the British govern- ment sought to reflect developments in reproductive technology and research since the act's inception in 1990. Among the recommendations (see www.dh.gov.uk/assetRoot/04/14/13/15/04141315.pdf) are new regulations on embryo research combining human and nonhuman material that take into account acute public concerns over where such research might lead.

This is set to ignite a debate about a central approach in the transition from basic research on stem cells to their clinical use, which might eventually reduce the need for human eggs and embryos in research. The onus is now on the scientific community to assume a proactive role in helping to define the boundaries of what is ethical and safe in interspecies research using human cells. At stake are both public support and the risk of delays to high-priority biomedical research.

In Britain, the governance of research combining cells, cellular material or genetic material from different individuals or species is nebulous. The new proposals provide a well-reasoned and permissive approach to the contentious issue of the nuclear transfer of human nuclei to a human donor egg for the purposes of therapeutic cloning, and also propose that other research combining animal and human materials should be subject to more stringent licensing procedures. The devil will be in the details, or lack of them, and will have policy implications for scientists working on cell-replacement therapies.

The document recognizes that human-animal fusion products have been widely used in biomedical research for many years (for example, in xenograft models of cancer in which human cells are introduced into mice), but also notes public unease with them. The lack of specific language in the document highlights a gap in the regulations that will need to be filled. How researchers will influence such policy decisions depends on their ability to swiftly agree and promote key research priorities, coupled to a clear assessment of how these priorities are balanced by both safety and ethical concerns.

\section{Current restrictions}

In the United States, the National Institutes of Health does not fund research involving the transplantation of human embryonic stem cells into animal embryos. In Canada, this funding restriction extends to the transplantation of human tissue-specific (or 'adult') stem cells to animal embryos. In 2005, the US National Academy of Sciences stated its opposition to research in which human embryonic stem cells are introduced into non-human primate blastocysts (pre-implantation embryos), or in which any embryonic stem cells are introduced into human blastocysts, as well as the breeding of any animal into which human embryonic stem cells have been introduced.

At present, such guidelines are reasonable but do not consider several promising and arguably necessary avenues of research that combine human cells or cellular components with other species. These include combining the genetic material of humans and other species, the prenatal combination of cells from different individuals (animal to human, human to animal, or human to human), or grafting tissue from humans to animals.

One troubling outcome of a debate could be UK restrictions on current work combining factors from animal eggs (which, unlike human eggs, are readily accessible) with animal or human nuclei. These 'nuclear reprogramming' experiments aim to identify components of the egg that are capable of transforming an adult cell into one with the vast capabilities of stem cells. They could generate stem cells and tissues genetically and immunologically matched to patients, and obviate the need for human eggs and embryos in generating human embryonic stem cells. There are strong arguments for permitting such research, given the minimal safety risk or violation of human dignity when any resulting embryos are arrested at an early stage.

\section{A question of balance}

Another important line of investigation is the engraftment of human stem cells into non-human primate models. Such work is essential, as it would be dangerous to have dinical trials for cell-replacement therapies in humans without first testing promising human cell lines in animal models, for example by transferring human neuronal cells into animal brains. A valuable and provocative discussion by Karpowicz et al. (Nature Med. 10, 331-335; 2004) outlines some key questions in balancing ethics with human benefits: how robustly the transplanted cells are incorporated into the host; at what stage and into what tissues and organs they are introduced; whether there is a possibility that introducing such cells would alter the production of sperm or eggs in the host animal; and, for neuronal transplantation, whether there is a risk of transferring human functions or behaviours to the host animal. This would be an unacceptable outcome whose risk, Karpowicz et al. argue, is generally negligible.

The avenues of research discussed here all fall under the rubric of combining animal and human material, and offer a high level of benefit, but with disparate degrees of risk, so they should be regulated differently. Scientists must provide recommendations on how to proceed, on where there is consensus, and where significant risks or ethical problems exist.

Scientists should identify the various research protocols defining interspecies research involving human cells and embryos, and the associated risks, ethical issues and benefits of each. They should put forward clear and comprehensive recommendations to the public and to regulatory bodies. If they don't, they risk having regulation and funding restrictions imposed on their research that are out of proportion to the ethical or safety risks involved. Even worse, they could face prohibitions that lump together research with vast disparities in intent and in the balance of risk and benefit - ultimately penalizing those who stand to gain from the therapies that might emerge. 